\title{
Circuit
}

Musiques contemporaines

Le son dans l'art contemporain canadien / Sound in

Contemporary Canadian Art, dirigé par / edited by Nicole

Gingras, Édition Artexte, Montréal, 2003, 238 pages

\section{Réjean Beaucage}

Volume 15, numéro 2, 2005

Cartes d'identités

URI : https://id.erudit.org/iderudit/902362ar

DOI : https://doi.org/10.7202/902362ar

Aller au sommaire du numéro

Éditeur(s)

Les Presses de l'Université de Montréal

ISSN

1183-1693 (imprimé)

1488-9692 (numérique)

Découvrir la revue

Citer ce compte rendu

Beaucage, R. (2005). Compte rendu de [Le son dans l'art contemporain canadien / Sound in Contemporary Canadian Art, dirigé par / edited by Nicole Gingras, Édition Artexte, Montréal, 2003, 238 pages]. Circuit, 15(2), 105-110. https://doi.org/10.7202/902362ar d'utilisation que vous pouvez consulter en ligne.

https://apropos.erudit.org/fr/usagers/politique-dutilisation/ 
Le son dans l'art contemporain canadien/Sound in Contemporary Canadian Art, dirigé par/edited by Nicole Gingras, Édition Artexte, Montréal, 2003, 238 pages.

Lorsque le 11 mars 1913 Luigi Russolo publia son manifeste L'Arte dei rumori (L'Art des bruits), il avait beau être futuriste, il ne se doutait pas une seconde de l'influence qu'il allait avoir sur les développements de ce qui a finalement pris le nom d'art sonore.

Rédigé comme une lettre adressée à son confrère futuriste Balilla Pratella, compositeur et lui-même auteur de quelques textes fondateurs (entre autres : Manifesto dei musicisti futuristi en 1910 et son développement La Musica futurista-Manifesto tecnico, en 1911), le texte de Russolo se termine par un paragraphe qui va littéralement ouvrir la boîte de Pandore :

Mon cher Pratella, je soumets à ton génie futuriste ces idées nouvelles en t'invitant à les discuter avec moi. Je ne suis pas un musicien. Je n'ai donc pas de préférences acoustiques ni des œuvres à défendre. Je suis un peintre futuriste qui lance hors de lui, sur un art profondément aimé, sa volonté de tout renouveler. C'est pourquoi, plus téméraire que le plus téméraire des musiciens de profession, nullement préoccupé par mon apparente incompétence, sachant que l'audace donne tous les droits et toutes les possibilités, j'ai conçu la rénovation de la musique par l'Art des Bruits'.

Et voilà! «Je ne suis pas musicien », mais j’ai des idées pour renouveler la musique... Russolo, préfigurant les déambulations urbaines des surréalistes, le «tout est musique» de John Cage et les soundwalks de Hildegard Westerkamp, nous lançait cette invitation :

Traversons ensemble une grande capitale moderne, les oreilles plus attentives que les yeux, et nous varierons les plaisirs de notre sensibilité en distinguant les glouglous d'eau, d'air et de gaz dans les tuyaux métalliques, les borborygmes et les râles des moteurs qui respirent avec une animalité indiscutable, la palpitation des soupapes, le va-et-vient des pistons, les cris stridents des scies mécaniques, les bonds sonores des tramways sur les rails, le claquement des fouets, le clapotement des drapeaux. Nous nous amuserons à orchestrer idéalement les portes à coulisses des magasins, le brouhaha des foules, les tintamarres différents des gares, des forges, des filatures, des imprimeries, des usines électriques et des chemins de fer souterrains? 2 .

Le renouvellement serait bientôt si total que, comme on ne retrouve plus, après avoir rangé ce que l'on trouvait si aisément quand tout était en désordre, les musiciens n'y reconnaîtraient plus la musique. Mais est-ce que ça en serait? Peut-on faire de la musique sans être musicien? Aussi bien demander, même avec un sourire en coin, si l'on peut faire de la littérature sans savoir écrire... Ou faire de la peinture... etc. Cette nouvelle musique qui n'en était plus avait besoin d'un nouveau nom. «Art des bruits » ou «bruitisme » auraient pu faire
1. Russolo, L. (1954) [1913], L'Art des bruits. Manifeste futuriste, Éditions Richard-Masse. 
3. À moins de le faire dans le cadre d'un acte d'écoute sciemment conçu comme tel, à la manière de la pièce 4' 33 "' de John Cage, qui fut par ailleurs inspirée au compositeur par la lecture du manifeste de Russolo (et qui n'est pas du tout à cet égard une mise en valeur du silence - dont Cage a pu prouvé l'inexistence lors d'une autre expérience - mais bien une mise en valeur du bruit). Comme le suggère Raymond Gervais dans son texte "Big Bang et postmodernité" (p. 152-165), l'intention peut aussi changer de côté : "Être présent, attentif aux sons de l'environnement, les écouter avec intérêt et les accepter peut-être en tant que musique à part entière, cela devient une question d'attitude, un choix individuel." On pourrait alors parler d'une façon artistique d'écouter, assimilable à l'expérience de voir des moutons dans les nuages.

4. Et on revient ici au paradoxe devant lequel nous place John Cage avec son exploration d'une musique "non intentionnelle" (et donc non musicale, la musique étant l'art d'organiser les sons). On pourrait en conclure que si les musiciens se lancent dans la nonmusique, il est peut-être normal que des non-musiciens fassent de la musique...

5. Nicole Gingras, dans le sommaire du livre, p. 10. l'affaire, mais ils seront réservés à la portion la plus tonitruante de ce nouvel art, qui sera, restons général, «sonore ».

Il est aujourd'hui devenu monnaie courante de voir des peintres, sculpteurs ou artistes «multi» de tout acabit accompagner leurs prestations publiques d'art sonore sous une forme ou une autre. Et comme si l'expression «art sonore» n'était pas encore assez englobante, Nicole Gingras préfère parler, comme l'indique le titre du livre dont il est question ici, du «son dans l'art contemporain » (qu'elle circonscrit quand même un peu en y ajoutant «canadien »). C'est sans doute le problème principal de cet ouvrage que d'annoncer un sujet qui demeure beaucoup trop vaste pour ses 238 pages si, en plus, on ne s'occupait pas d'aller chercher «le son artistique » là où il n'est pas... Doit-on vraiment s'émouvoir, comme le fait l'auteure, devant le bruit que font les pages d'un livre d'artiste lorsqu'on les toume? Ou en écoutant le «son silencieux» (unsound) produit durant une exposition n'incluant aucun dispositif sonore, comme le fait Christof Migone?

Comme Marcel Duchamp devait signer ses ready-made pour les élever au rang d'œuvre d'art, il faudrait au moins qu'un artiste ait la volonté d'inclure du «sonore» dans son œuvre pour que l'on prétende en discuter. Nous vivons à chaque milliseconde dans une "photographie potentielle", mais il faut quand même que quelqu'un fasse «clic»! L'intention est l'élément qui transforme la matière première en «Art». Or, on perpétue dans ce livre le malentendu déjà présent dans la citation de Luigi Russolo : écouter la rumeur de la ville (ou le murmure de la forêt), ce n'est pas écouter une manifestation artistique ${ }^{3}$; mais choisir « [d']orchestrer idéalement les portes à coulisses des magasins, le brouhaha des foules, [...]", c'est faire de la musique. Il y a donc dans ce livre beaucoup de musique qui ne sait pas qu'elle en est et qui, mais oui, est faite par des non-musiciens ${ }^{4}$. Bon, mais qu'y a-t-il, au juste, dans ce livre? On y vient.

\section{Le livre et le CD}

Élaboré dans le cadre d'une résidence de l'auteure au Centre d'information en art contemporain Artexte, le livre se présente comme un recueil de textes disparates (réflexions théoriques, palmarès historiques, descriptions de projets) regroupés dans le but de documenter les nouvelles pratiques artistiques, incluant le son, qui ont été développées (surtout) dans la seconde moitié du $\mathrm{XX}^{\mathrm{e}}$ siècle. L'auteure voulait principalement «[s]ouligner l'apport du son et du sonore dans les arts visuels et médiatiques au Canada ainsi qu'identifier un mode exploratoire de pensée et de création à la jonction de la musique, des arts visuels et du performatif " ". On note tout de suite l'utilisation, dans la même phrase, des termes «son» (ou «sonore») et «musique», dont les définitions 
ne seront jamais clairement établies tout au long de l'ouvrage. Et ce n'est pas en mettant sur le même pied tout ce qui est susceptible d'entrer par les oreilles (sans parler des «sons intérieurs» [inner speech] de Christof Migone), qu'on le fera. Le livre ne cherche donc pas à identifier des différences entre ce qui est «musique » et d'autres «manifestations sonores ", mais prend plutôt les allures d'un catalogue non exhaustif, et finalement assez désordonné, des pratiques artistiques les plus diverses (excluant, tout de même, le cinéma, le théâtre ou la danse) dans lesquelles l'utilisation du «son » n'est quelquesfois qu'un paramètre négligeable.

Dans son texte «Résonances », qui est en quelque sorte une seconde préface, Nicole Gingras présente quatre «expériences d'écoute » qui ont plus ou moins servi de déclencheurs au projet. Il s'agit d'un environnement vidéo (Impression débâcle, de Michèle Waquant, 1992, Musée d'art contemporain de Montréal), d'une exposition (Kate Craig : Skin, 1998, Vancouver Art Gallery), d'une sculpture (Le jardin des Ovelyniers, de Nicolas Reeves, 1999-2001, L'art qui fait boum) et d'une performance (The Disconnection Machine, de Nerve Theory, 2001, Festival Elektra); cet échantillonnage restreint, s'il donne une bonne idée de la pluralité des approches examinées ici, pose encore une fois le problème de définition évoqué plus haut. On est dans le monde des espaces d'exposition, galeries ou musées, mais s'y glisse pourtant Elektra, un événement consacré aux arts numériques qui est pratiquement un festival musical. Si l'œuvre de Michèle Waquant recourt à une bande sonore constituée de « voix, extraits de la radio (bulletin météo, musique populaire), bruits et craquements de la glace, souffles du vent ${ }^{6}$ », la performance de Tom Sherman est un «flux de paroles, de sons et d'images vidéo repiquées de l'Internet» auquel participe le musicien Bernhard Loibner et « [c]e qui étonne dans The Disconnection Machine, c'est justement la rencontre entre le langage, la performance, la vidéo et la musique $^{7} »$. Les musiques populaires utilisées par Waquant ont un rôle décoratif tandis que dans le deuxième cas, la musique participe à l'œuvre. Dans celui de la sculpture sonore de Nicolas Reeves, il s'agit de cinq oranges disposées sur un lit de cristaux au fond d'un aquarium et recouvertes d'eau.

Dans chaque orange sont plantées deux électrodes, une en cuivre et une en zinc, qui collectent en sortie, grâce à l'acidité du fruit, une tension d'environ 0,7 volt par orange. [...] Le courant électrique est ensuite amplifiée [et] contrôle l'émission de séquences sonores, diffusées par quatre enceintes acoustiques [...]. Les sons émis résultent de l'hybridation d'échantillons de voix humaines et de séquences rythmiques obtenues, construites, par synthèse granulaire ${ }^{8}$.

On pourrait arguer que, bien que ce nouvel instrument de musique soit vivant et périssable, les sons déclenchés par les oranges n'ont rien de fortuit, et
6. p. 19.

7. p. 21.

8. Nicolas Reeves, dans son texte "Le jardin des Ovelyniers ou La lanterne aux oranges méditantes", p. 136. 
9. Note 4, p. 21.

10. p. 20.

11. p. 26.

12. p. 35 .

13. Jocelyn Robert, "L'enregistrement universel continu", p. 64. que Reeves y a mis assez d'intention pour que l'on puisse en effet parler de musique. En revanche, on ne saura jamais de quoi est faite la bande sonore accompagnant l'exposition de Kate Craig car, même si «[d]ans toutes les vidéos de Craig, le son joue un rôle déterminant ${ }^{9}$ ", tout ce que l'on en saura, c'est qu'elle «crée $[. .$.$] un étonnant paysage sonore, fait de retours, d'appels, d'asso-$ ciations, d'évocations et d'impressions ${ }^{10} " .$. La réflexion qui aurait pu être développée sur la différence entre le «sonore» et le «musical», et sur les raisons pour lesquelles on les mélange aussi allégrement ici, ne viendra malheureusement pas.

Nicole Gingras rédige aussi "Dispositifs», un texte qui se veut une "réflexion sur les modes et modalités de documentation, de diffusion et de création des œuvres ${ }^{11}$ ». L'auteure y fait l'inventaire de certaines manifestations artistiques (expositions, performances, etc.) qui ont laissé des traces chez Artexte. Il s'agit en quelque sorte d'amplifier ces traces en les répertoriant, d'offrir une certaine pérennité à des créations qui tiraient souvent leur intérêt de leur caractère éphémère, bref de donner ses lettres de noblesse à une forme d'art largement underground. On trouve par ailleurs dans cette liste un disque (Ding Dong Deluxe, Avatar, 1994) qui n'est pas autre chose qu'un disque et qui ne se rattache à aucune autre forme de manifestation. On trouve aussi une série de concerts (Volt AA, organisée par Eric Mattson, pompeusement titré "commissaire») qui, bien qu'elle offre certaines particularités intéressantes, présente tout de même des artistes faisant entendre de la musique à un public (et le fait que "l'écoute [y soit] favorisée et le silence, de rigueur ${ }^{12}$ » ne saurait sérieusement être présenté comme une nouveauté dans le cadre d'un concert...). Un texte, donc, qui présente en concentré le malaise qui caractérise le livre : le refus de choisir, ou l'incapacité de faire la différence, entre «musique» et «art sonore».

Après ces textes de présentation de Nicole Gingras, le livre accueille diverses collaborations réunies en sections. D'abord, «Penser le son/Thinking Through Sound» qui s'ouvre sur un court texte de Jocelyn Robert qui réfléchit sur le fait que «si on pouvait regarder de très près chacune des molécules qui constituent la paroi d'une poterie ancienne, on pourrait reconstituer le chant de la travailleuse qui l'a fabriquée ${ }^{13}$ ", une théorie qui, malgré son intérêt, ne semble pas à sa place ici. La section se poursuit par un texte de R. Murray Schafer qui n'est certes pas l'un de ses meilleurs... L'auteur y décline une enfilade de trivialités présentées comme de "grandes vérités», afin de nous faire comprendre que le son a une certaine importance dans nos vies (There is no silence for the living. [...] Sound gets to place where sight cannot. [...] I am always at the heart of thesounding universe. Etc.). Vient ensuite un texte de Raymond Gervais qui 
rappelle l'importance du compositeur Pierre Mercure dans le développement des «musiques nouvelles», principalement par son intérêt pour l'électroacoustique et, bien sûr, par l'organisation en 1961 de la Semaine internationale de musique actuelle, qui permit aux mélomanes montréalais de l'époque de découvrir les musiques de John Cage, Morton Feldman, Christian Wolff, Serge Garant, Edgar Varèse, et d'autres. Cette Semaine accordait par ailleurs une grande importance aux arts visuels (danse, costumes, décors, éclairages, projections, sculptures), qui nous permet d'observer un premier point de jonction entre la musique et les arts visuels chez nous.

La section suivante, "Volumes et surfaces/Volumes and Surfaces », s'intéresse aux propriétés physiques du son. Christof Migone y aborde la densité du son dans l'espace, réussissant à mettre sur le même pied l'installation de 400 hautparleurs par Iannis Xenakis dans le Pavillon Philips (Exposition universelle de Bruxelles, 1958) et une installation de Michael Fernandes n'incluant aucun dispositif sonore (I Am a Failure : Room of Fears, 2000); Gordon Monahan y explique la genèse de sa performance Speaker Swinging (1981), qui explore l'effet Doppler ${ }^{14}$; Michèle Waquant présente son installation Impression débâcle; le concepteur sonore Colin Griffith s'intéresse aux propriétés psychoacoustiques des lieux de diffusion; enfin, Hildegard Westerkamp rend compte d'une expérience d'écoute active réalisée en Inde en 1998. La section «Espaces d'écoute/Listening Spaces» offre d'autres exemples d'utilisation du son (musical ou non) : Steve Heimbecker décrit son installation Wind Array Cascade Machine (2003), qui utilise les mouvements du vent pour déclencher des effets visuels et sonores (il est intéressant de constater que pour ce dernier volet de l'œuvre, l'artiste utilise le terme « composition»); Gayle Young décrit une exposition d'instruments de musique inventés (Sounds of Invention, Memorial University Art Gallery, 1991); Nicolas Reeves explique les origines et le fonctionnement de son assemblage Le Jardin des Ovelyniers; enfin, la réalisatrice radiophonique Hélène Prévost présente trois «événements reposant sur des dispositifs originaux ${ }^{15}$ »... et hautement musicaux : Le $7^{e}$ Printemps électroacoustique (1992), les Symphonies portuaires (1995) et le Silophone (2000).

La dernière section du livre, "Agencements : corps installation, corps machine/Modulations : Installation Body and Machine Body", offre encore d'autres exemples d'utilisations du son dans le cadre de performances ou d'installations.

Le disque compact qui accompagne le livre se révèle utile pour avoir une meilleure idée du résultat sonore de quelques-unes des expériences décrites dans le livre. Il contient des œuvres musicales (Hildegard Westerkamp, Martin Tétreault, Bill Mullan, Georges Azzaria, Jean Routhier), des enregistrements
14. Voir notre propre compte rendu d'une représentation de Speaker Swinging dans "Elektra 2002 : le poids du passê", Circuit musiques contemporaines, vol. $3 n^{\circ} 3$, p. $82-83$.

15. Hélène Prévost, "Écoutes urbaines", p. 141. 
documentant des installations (Jean-Pierre Gauthier, Michael Snow, Ken Gragory, Diane Landry, Rita McKeough) ou des expériences sonores diverses (Pierre-André Arcand, Rober Racine, Hugh Le Caine, Daniel Olson, Emmanuel Madan).

On peut faire plusieurs reproches à ce livre, dont le plus sérieux est son manque de rigueur dans le choix des sujets traités (le problème musique/ art sonore, jamais abordé). Cependant, on peut aussi le considérer comme très utile pour documenter de nombreuses activités artistiques, vouées à l'oubli sans l'intervention de l'auteure. Et le reproche principal qu'on lui adresse n'est à vrai dire que relatif à une mise en place de la réflexion pour laquelle ce livre aura servi de déclencheur, et qui reste à faire.

Il est à noter que l'on peut aussi compléter la lecture de ce livre et l'écoute du disque qui l'accompagne par celle d'un autre disque compact réalisé par Nicole Gingras et réunissant les bandes sonores des installations formant l'exposition Frottements. Objets et surfaces sonores, présentée au Musée national des beaux-arts du Québec du 2 septembre au 28 novembre 2004. L'exposition, pour laquelle Nicole Gingras agissait à titre de commissaire, réunissait des œuvres de Diana Burgoyne, Jean-Pierre Gauthier, Raymond Gervais (dont l'œuvre muette n'est évidemment pas représentée sur le disque), Erika Lincoln, Daniel Olson, Rober Racine, Jocelyn Robert, Martin Tétreault et du duo skoltz_kolgen. 